tive amplification of RNA molecules with new, predetermined activities.

The idea behind attempts to select RNAs with particular properties is simple: in essence, it is the equivalent of evolutionary selection at the level of individual molecules. Thus, starting with a set of variants on the ribozyme derived from the self-splicing intron of Tetrahymena precursor ribosomal RNA, Robertson and Joyce have selectively amplified RNAs according to their ability to catalyse the cleavage of a DNA substrate (Nature 344, $467-468 ; 1990)$. Robertson and Joyce devised a scheme in which the ability of the catalyst to cleave DNA was linked to copying of the RNA molecule into DNA by reverse transcriptase (see figure). In this way, they selected variants with increased ability to cleave DNA from a set of deletion mutants of the 'wild-type' ribozyme.

A similar approach has been used to explore the sequence requirements for activity at certain positions of the same Tetrahymena ribozyme. Rachel Green (Massachusetts General Hospital, Boston) reported how a ligation reaction catalysed by the ribozyme was arranged to create a substrate for reverse transcription into complementary DNA and then amplification by the polymerase chain reaction (PCR). A set of roughly 250,000 variants of the wild-type ribozyme was made at nine sites chosen by phylogenetic sequence comparison. From this set RNAs were selected with catalytic activity at least as great as that of the starting wild-type RNA. The sequences of these RNAs suggest that complex interactions crucial to the function of the ribozyme occur between certain combinations of the varied bases.

To expand the repertoire of reactions that can be catalysed by ribozymes, a good starting point would be a set of RNAs that can selectively bind novel ligands. To obtain such molecules, Andrew Ellington (Massachusetts General Hospital, Boston) has linked the replication of RNA to its ability to bind to an affinity chromatography column. Ellington started by synthesizing random sequence DNA, which is linked to sequences that allow the DNA to be transcribed into RNA, and at a later stage the RNA to be copied back into DNA.

Transcription of the random sequence DNA, of average length 100 bases, gave a yield of RNA expected to contain about $10^{15}$ different sequences. This RNA was passed over columns containing specific dye molecules. The RNA that bound to the column was retained and copied by reverse transcription into DNA. This was amplified by PCR, and then the whole cycle was repeated. After five rounds, most of the selected RNA had bound to the dye used to select it, and not to the

* The 1990 Cold Spring Harbor RNA processing meeting. 16-20 May.

\title{
A long and ancient road
}

IN a two-part report in this month's issue of Antiquity $(64,210-220,1990)$ Hillam et al. announce an exact date for the Neolithic timber pathway known as the Sweet Track. The track, one of the oldest known man-made structures in Europe, was constructed from timber felled in the winter and early spring of 3807-3806 BC. Although the track

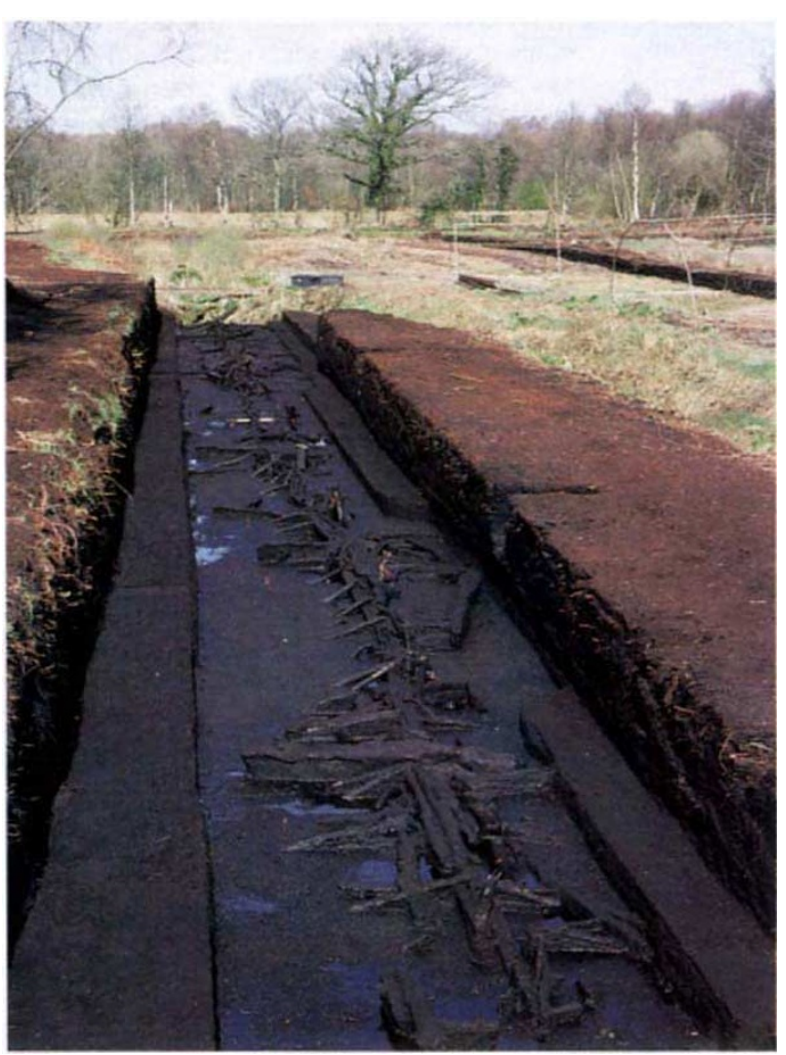
had aiready been dated approximately by radiocarbon, the authors pinned down its precise age by matching tree rings in the preserved timbers to a 'master' 7,000-year chronology of oaks from Irish and German bogs.

In the second part of the report, Coles and Coles discuss the track itself (some of the remains of which can be seen in the picture) and the archaeological context. It consisted of a single line of planks, about 1,800 metres in length, which ran across a swamp in what is now the British county of Somerset. Some of the wood used was felled slightly later than the rest, implying that the track underwent a series of repairs a few years after it was built. From the dating of these repairs, the authors conclude that the track could have been in use only for about ten years, before being overwhelmed by the swamp.

A range of artefacts and other remains recovered from the peat, either alongside or beneath the track, can therefore be linked with a precise time, allowing us a glimpse of some of the activities of Stone Age men and women and the environment in which they lived. For example, from analysis of beetle remains found near the track it seems that British winters were $2-4{ }^{\circ} \mathrm{C}$ colder and summers $2-3^{\circ} \mathrm{C}$ warmer than they are today.

Among the artefacts recovered are potsherds, a tomahawk, a comb, toggles, a spoon and even a piece of grass rope. Of particular note are two axeheads preserved in practically mint condition - one made of jadeite, the other of flint. The flint has been identified as coming from the flint mines in Sussex, whereas the jadeite is thought to be from the Swiss Alps. Clearly, some kind of European marketing system was in use even then.

Philippa Lloyd

other, chemically quite similar dyes. Some of the selected RNAs have been sequenced, and RNAs independently selected by a particular dye turn out to have only limited sequence similarity. This may be significant - mutagenesis of two of the selected RNAs showed the sites in the RNAs that are crucial for dyebinding, and it turns out that there is a good correspondence between these sites and sites where the sequences of some of the independently selected RNAs are identical. The implication is that the same structural solution to the problem of binding specific dye molecules has been found more than once in the selection procedure

Two of the dyes used in these experiments are structurally similar to the bio- logical cofactor nicotinamide adenine dinucleotide (NAD), and starting from RNAs selected to bind these dyes Ellington has been able to apply the same procedure to select for binding to NAD. He intends to go on and select for RNAs that bind structural analogues of the transition states for particular chemical reactions, in the hope that the RNA will turn out to catalyse the reaction, and in this way to develop novel ribozymes.

An alternative approach to the synthesis of RNA enzymes with new catalytic activities would be that of rational design based on knowledge of the structure of an existing ribozyme. In no case so far is the full three-dimensional structure of a ribozyme known, but A. Pardi (University of Colorado, Boulder) reported progress in 